\title{
Finanzierung medizinischer Leistungen in Haft
}

Sehr geehrte Damen und Herren

Die Verbindung der Schweizer Ärztinnen und Ärzte (FMH) und die Schweizerischen Gefängnisärzte (KSG) sind beunruhigt, weil der Zugang zu medizinischen Leistungen im Strafvollzug zunehmend an eine finanzielle Beteiligung der inhaftierten Personen geknüpft ist. Im Februar 2019 veröffentlichte die Zentrale Ethikkommission der Schweizerischen Akademie der Medizinischen Wissenschaften SAMW eine Stellungnahme zur Finanzierung medizinischer Leistungen im Gefängnis. Darin weist sie auf die Bedeutung einer effektiven und qualitativ hochwertigen medizinischen Versorgung hin, die jener der Allgemeinbevölkerung gleichwertig ist und unabhängig vom Aufenthaltsstatus gewährleistet werden soll.

Die SAMW-Richtlinien zur Ausübung der ärztlichen Tätigkeit bei inhaftierten Personen halten fest, dass die medizinische Versorgung niederschwellig zugänglich und grundsätzlich kostenfrei sein sollte, abgesehen von einer leistbaren und angemessenen Kostenbeteiligung. Diese ist indes in der Realität nur in Ausnahmefällen möglich.

Ärztinnen und Ärzte in verschiedenen Institutionen des Freiheitsentzugs beobachten seit längerem, dass der Zugang zu medizinischen Leistungen komplizierter wird. Sie müssen zusätzliche Energie und Zeit mit Kostenübernahmegesuchen und Verhandlungen mit administrativen Stellen bei Justizvollzugs- oder Sozialämtern aufwenden, um ihren Patientinnen und Patienten eine angemessene Gesundheitsversorgung zu ermöglichen. Kürzlich wurde in einem Kanton beschlossen, dass Rechnungen nicht mehr an die Vollzugsbehörde geschickt werden dürfen. Diese sollen direkt dem inhaftierten Patienten zugestellt und erst nach drei unbezahlten und dokumentierten Mahnungen der Vollzugsbehörde weitergeleitet werden.

Andere Kantone haben dieses Jahr begonnen, Gesundheitsleistungen direkt der inhaftierten Person in Rechnung zu stellen, unabhängig vom Versicherungsstatus oder ihrer - im Regelfall prekären - finanziellen Situation. Das Einkommen einer inhaftierten Person liegt im Durchschnitt bei weniger als 500 Franken pro Monat, vorausgesetzt, sie bekommt überhaupt Zugang zu bezahlter Arbeit.

Die Gefängnismedizin kann somit wichtige Funktionen für die öffentliche Gesundheit nicht mehr wahrnehmen. Auch wird grundsätzlich die Qualität der Gefängnismedizin gemindert. Wie soll ein Arzt oder eine Ärztin Infektionskrankheiten wie Tuberkulose, HIV oder Hepatitis C erkennen, wenn inhaftierte Personen aus Angst vor einer finanziellen Mitbeteiligung nicht zum Arzt oder zur Ärztin kommen oder - wie inzwischen vielfach beobachtet - auf geplante Untersuchungen und Behandlungen verzichten?

Wenn Krankheiten nicht mehr rechtzeitig erkannt und behandelt werden, können Personen - auch nach Haftentlassung - eine Gesundheitsgefahr für die Gesellschaft darstellen.

Medizinische Leistungen in Haft müssen äquivalent sein zu denjenigen der Allgemeinbevölkerung in Freiheit. Es darf keine Rolle spielen, ob die inhaftierte Person dem KVG unterstellt ist oder nicht. Der Leistungskatalog gemäss KVG darf nicht unterschritten werden. Heute haben die Kantone unterschiedliche Systeme zur Kostenträgerschaft, was eine Ungleichbehandlung darstellt. Es sollte eine schweizweit einheitliche Lösung erarbeitet werden. Wünschenswert wäre, künftig alle inhaftierten Personen dem Krankenversicherungsobligatorium zu unterstellen.

\section{Mit freundlichen Grüssen}

Prof. Hans Wolff, Präsident KSG

Dr. Jürg Schlup, Präsident FMH

\section{Literatur}

1 Stellungnahme der Zentralen Ethikkommission der SAMW (2019). Finanzierung medizinischer Leistungen im Gefängnis. www.samw.ch/de/Publikationen/Stellungnahmen.html

2 NKVF: Gesamtbericht über die schweizweite Überprüfung der Gesundheitsversorgung im Freiheitsentzug. www.nkvf.admin.ch/ nkvf/de/home/publikationen/newsarchiv/2019/2019-11-14.html 